\title{
Combining canonical correlation analysis and infinite reference for frequency recognition of steady-state visual evoked potential recordings: A comparison with periodogram method
}

\author{
Yin Tian ${ }^{\mathrm{a}, \mathrm{b}, \mathrm{c}, *}$, Fali $\mathrm{Li}^{\mathrm{a}, \mathrm{c}}$, Peng Xu ${ }^{\mathrm{c}}$, Zhen Yuan ${ }^{\mathrm{b}, *}$, Dechun Zhao and Haiyong Zhang ${ }^{\mathrm{a}}$ \\ ${ }^{a}$ Bio-Information College, Chongqing University of Posts and Telecommunications, Chongqing \\ 400065, China \\ ${ }^{b}$ Bioimaging Core, Faculty of Health Sciences, University of Macau, Macau SAR, China \\ ${ }^{c}$ Key Laboratory for NeuroInformation of Ministry of Education, School of Life Science and \\ Technology, University of Electronic Science and Technology of China, Chengdu 610054, China
}

\begin{abstract}
Steady-state visual evoked potentials (SSVEP) are the visual system responses to a repetitive visual stimulus flickering with the constant frequency and of great importance in the study of brain activity using scalp electroencephalography (EEG) recordings. However, the reference influence for the investigation of SSVEP is generally not considered in previous work. In this study a new approach that combined the canonical correlation analysis with infinite reference (ICCA) was proposed to enhance the accuracy of frequency recognition of SSVEP recordings. Compared with the widely used periodogram method (PM), ICCA is able to achieve higher recognition accuracy when extracts frequency within a short span. Further, the recognition results suggested that ICCA is a very robust tool to study the brain computer interface (BCI) based on SSVEP.
\end{abstract}

Keywords: Steady-state visual evoked potentials, canonical correlation analysis with infinite reference, frequency recognition, periodogram, brain computer interface

\section{Introduction}

Steady-state visual evoked potentials (SSVEPs) are periodic evoked potentials induced by the repetitive visual stimulus flickering with the constant frequency over $4 \mathrm{~Hz}[1,2]$. SSVEP is usually recorded by scalp electroencephalography (EEG) with high temporal resolution and has been extensively utilized in the study of cognitive neurosciences, neurological disorders and BCI [2]. However, how to

\footnotetext{
${ }^{*}$ Corresponding authors: Yin Tian, Bio-information College, Chongqing University of Posts and Telecommunications, Chongqing 400065, China. Tel./Fax: 86-23-62460536; E-mail: YinTian2010@gmail.com.

Zhen Yuan, Bioimaging Core, Faculty of Health Sciences, University of Macau, Macau SAR, China. Tel./Fax: 85388224989/88222314; E-mail: Zhenyuan@umac.mo.
} 
quickly and effectively identify the brain SSVEP signal corresponding to the change of external stimuli and how to successfully realize real-time signal processing are still one of the biggest challenges we might meet in the study of SSVEP-based BCI.

To resolve these issues, several algorithms for frequency recognition of SSVEP recordings have been implemented including the power spectral density analysis (PSDA) and canonical correlation analysis (CCA) [3-6]. PSDA has been well established, which utilizes the limited length of data to estimate the signal power spectrum. In particular, PM is the mostly adopted algorithm for the fundamental analysis in PSDA. In contrast, CCA employs the multivariable statistical analysis for extracting maximum similarity between two data sets, which has also been successfully applied to frequency recognition of SSVEP data processing [6].

However, scalp EEG recordings are prone to be affected by the non-zero reference choice [7-9], which will further the influence on EEG waveform, power spectrum, and EEG brain network [9-12]. Therefore, the choice of an ideal reference such as infinity reference (IR) with zero potential is an essential step for EEG recordings. In this study, a novel method called canonical correlation analysis with infinite reference (ICCA) was proposed and implemented to improve the accuracy and validity of frequency recognition of SSVEP data processing. To the best of our knowledge, this is the first investigation of EEG-based BCI that is influenced by the choice of reference.

\section{Material and methods}

\subsection{Subjects and EEG recordings}

Five normal right-handed male subjects aged 20-26 participated in the experiments. None of them has been reported to have any history of mental or neurological problems. Informed consent was signed prior to the study, and subjects also received a monetary compensation. All experiments were approved by the ethical committee of Chongqing university of Posts and Telecommunications. A 64channel NeuroScan system was used for EEG data acquisition with a vertex reference. The EEG recordings were sampled with frequency $1000 \mathrm{~Hz}$ whereas all impedances were kept below $5 \mathrm{k} \Omega$ during the experiment.

120-second resting-state data was recorded. Then five frequencies, i.e. ten, twelve, fifteen and twenty $\mathrm{Hz}$, presented random to acquire SSVEP signals for 60 seconds. The configuration of the trigger was a $2 \times 2 \mathrm{~cm}^{2}$ square and the duty cycle is point five, point five, point six, point five and point five for the 5 frequencies, respectively [13]. A notebook with refresh rate of sixty Hertz was utilized here to present the triggers. Subjects sat about sixty centimeters away from the center of the monitor and were requested to gaze binocularly for all the flickering stimuli. The experiment duration was about one hour. To minimize the influence of signal excursion, EEG signals were firstly filtered with 1-100 $\mathrm{Hz}$ and subsequently resampled to $250 \mathrm{~Hz}$. For each subject, we chopped down the data sets and chose epochs (trial duration: 30s) with any artifacts from the SSVEP recordings for further analysis

\subsection{Canonical correlation analysis combined with infinite reference (ICCA)}

After pre-processing, the EEG data was re-referenced to the infinity reference (IR) using software REST [8,9] and then CCA method [6] was further employed for frequency recognition analysis. CCA is a statistical analysis method that can investigate the integration correlation between two sets of simi- 
lar recordings $X$ and $Y$. The key steps of the classical CCA are to find a linear combination $W_{x}$ and $W_{y}$ from $X$ as well as $Y$,

$$
\left\{\begin{array}{l}
x=x_{1} W_{x 1}+x_{2} W_{x 2}+\cdots+x_{p} W_{x p}=X^{T} W_{x} \\
y=y_{1} W_{y 1}+y_{2} W_{y 2}+\cdots+y_{q} W_{y q}=Y^{T} W_{y}
\end{array}\right.
$$

The maximized value of the correlation coefficients between two variances was obtained via an optimization procedure using the following equation,

$$
\max \rho(x, y)=\frac{W_{\mathrm{x}}^{T} C_{x y} W_{y}}{\sqrt{\left(W_{x}^{T} C_{x x} W_{x}\right)\left(W_{y}^{T} C_{y y} W_{y}\right)}}
$$

With the constraint conditions,

$$
\left\{\begin{array}{l}
W_{x}^{T} C_{x x} W_{x}=1 \\
W_{y}^{T} C_{y y} W_{y}=1
\end{array}\right.
$$

in which $C_{x x}$ and $C_{y y}$ are the corresponding covariance matrices of $X, Y$, respectively. And $\mathrm{C}_{\mathrm{xy}}$ is the cross-covariance matrix between $X$ and $Y$.

The minimal new paired variable sets can be observed by CCA. The observed new paired variables are not correlated with one another and have the capabilities of keeping the most relevant information in their original variables. Compared with the simple correlation analysis and multiple regression analysis, CCA method is the further development of principal component analysis (PCA) and factor analysis (FA) methods. CCA can test the correlation between two original variable sets, showing a many-to-many rather than a one-to-one relationship.

\subsection{Periodogram method (PM)}

After FFT of signal sequence $x(n)$, we would find both the generated frequency signal $X(w)$ and associated power spectrum would have periodic characteristics. The power spectrum analysis method used here is PM [14], which is a nonparametric method for signal power spectral density analysis. The advantages of PM are that it can be estimated by FFT and implement the correlation analysis of signals,

$$
X\left(e^{j w}\right)=\sum_{n=0}^{N-1} x(n) e^{-i w n}
$$




$$
S_{N R}(w)=\frac{1}{N}\left|X\left(e^{j w}\right)\right|^{2}
$$

in which $N$ is the sample number for spectrum estimation.

Compared to the parameter methods such as AR model, maximum likelihood spectrum estimation and feature decomposition method, etc., PM doesn't need estimate autocorrelation function and is able to achieve better estimation of power spectrum identification analysis.

\section{Results}

\subsection{Comparison analysis}

In consideration of the drawbacks of PM, we chop up the 30s SSVEP recordings equally into 6 epochs with 1250 sample points per epoch. As such, 18 data sets are generated for each subject with three specific frequencies 10,12 and $15 \mathrm{~Hz}$.

Frequency recognition accuracy was defined by the correct number and the corresponding SSVEP data,

$$
\text { Accuracy }=\frac{O}{K}
$$

in which Accuracy is the recognition accuracy, $O$ is the number of the right frequency recognition and $K$ is the total number of stimulus frequency.

The recognition results from both ICCA and PM methods are provided in Table 1, in which we can see that the recognition accuracy from ICCA is $15.56 \%$ higher than that from PM (paired t-test: $p<0.05$ ). As a result, ICCA is more suitable for frequency recognition of SSVEP recordings.

Table 1

Comparison for ICCA and PM

\begin{tabular}{llllll}
\hline Subject & ICCA & & & PM & \\
\cline { 2 - 3 } \cline { 5 - 6 } Name & $\begin{array}{l}\text { Correct } \\
\text { Count }\end{array}$ & $\begin{array}{l}\text { Recognition } \\
\text { Accuracy } \\
(\%)\end{array}$ & & $\begin{array}{l}\text { Correct } \\
\text { Count }\end{array}$ & $\begin{array}{l}\text { Recognition } \\
\text { Accuracy } \\
(\%)\end{array}$ \\
\hline LX & 18 & 100 & & 94.44 \\
ZY & 17 & 94.44 & 17 & 77.78 \\
CL & 18 & 100 & 13 & 72.22 \\
LH & 16 & 88.89 & 13 & 72.22 \\
LK & 18 & 100 & 16 & 88.89 \\
average & 17.6 & 96.67 & 14.6 & 81.11 \\
\hline
\end{tabular}


Table 2

Quantitative analysis using ICCA and PM

\begin{tabular}{|c|c|c|c|c|c|c|c|c|c|c|c|}
\hline \multirow{2}{*}{$\begin{array}{l}\text { Sub- } \\
\text { ject }\end{array}$} & \multirow{2}{*}{$\begin{array}{l}\text { Me- } \\
\text { thods }\end{array}$} & \multicolumn{2}{|l|}{$1 \mathrm{~s}$} & \multicolumn{2}{|l|}{$2 \mathrm{~s}$} & \multicolumn{2}{|l|}{$3 \mathrm{~s}$} & \multicolumn{2}{|l|}{$5 \mathrm{~s}$} & \multicolumn{2}{|l|}{$10 \mathrm{~s}$} \\
\hline & & $\begin{array}{l}\text { Cor- } \\
\text { rect } \\
\text { Count }\end{array}$ & $\begin{array}{l}\text { Accura- } \\
\text { cy (\%) }\end{array}$ & $\begin{array}{l}\text { Cor- } \\
\text { rect } \\
\text { Count }\end{array}$ & $\begin{array}{l}\text { Accura- } \\
\text { cy (\%) }\end{array}$ & $\begin{array}{l}\text { Cor- } \\
\text { rect } \\
\text { Count }\end{array}$ & $\begin{array}{l}\text { Accura- } \\
\text { cy (\%) }\end{array}$ & $\begin{array}{l}\text { Cor- } \\
\text { rect } \\
\text { Count }\end{array}$ & $\begin{array}{l}\text { Accura- } \\
\text { cy }(\%)\end{array}$ & $\begin{array}{l}\text { Cor- } \\
\text { rect } \\
\text { Count }\end{array}$ & $\begin{array}{l}\text { Accura- } \\
\text { cy (\%) }\end{array}$ \\
\hline \multirow{2}{*}{ LX } & ICCA & 83 & 92.33 & 45 & 100 & 30 & 100 & 18 & 100 & 9 & 100 \\
\hline & $\mathrm{PM}$ & 24 & 26.67 & 24 & 53.33 & 22 & 73.33 & 17 & 94.44 & 9 & 100 \\
\hline \multirow{2}{*}{ ZY } & ICCA & 87 & 96.67 & 45 & 100 & 30 & 100 & 17 & 94.44 & 9 & 100 \\
\hline & PM & 20 & 22.22 & 17 & 37.78 & 15 & 50 & 14 & 77.78 & 8 & 88.89 \\
\hline \multirow{2}{*}{ CL } & ICCA & 75 & 83.33 & 44 & 97.78 & 30 & 100 & 18 & 100 & 9 & 100 \\
\hline & PM & 19 & 21.11 & 18 & 40 & 19 & 63.33 & 13 & 72.22 & 8 & 88.89 \\
\hline \multirow{2}{*}{ LH } & ICCA & 49 & 54.44 & 27 & 60 & 23 & 76.67 & 16 & 88.89 & 8 & 88.89 \\
\hline & $\mathrm{PM}$ & 11 & 12.22 & 6 & 13.33 & 12 & 40 & 13 & 72.22 & 6 & 66.67 \\
\hline \multirow{2}{*}{ LK } & ICCA & 89 & 98.89 & 45 & 100 & 30 & 100 & 18 & 100 & 9 & 100 \\
\hline & PM & 25 & 27.78 & 21 & 46.67 & 20 & 66.67 & 16 & 88.89 & 8 & 88.89 \\
\hline
\end{tabular}

\subsection{Quantitative analysis}

The SSVEP recordings are segmented into different data sets in terms of 250, 500, 750, 500, 2500 sampling points per epoch. Altogether we have 90, 45, 30, 18, 9 data sets for the 3 frequencies. ICCA is utilized for quantitative analysis and the analysis results are given in Table 2. We can see from Table 2 that to a certain extent, the data length will affect the validity of ICCA. For example, recognition accuracy is decreased with decreased sampling points per epoch and increased with increased sampling points per epoch.

In PM, original data sets for each subject are segmented in terms of $1 \mathrm{~s}, 2 \mathrm{~s}, 3 \mathrm{~s}, 5 \mathrm{~s}, 10 \mathrm{~s}$ time window length per epoch. Power spectrum of each epoch will be calculated respectively. Figure 1 shows that for PM, the time window length has significant influences on the accuracy of frequency recognition of SSVEP recordings. The power spectral resolution will not meet the requirements of frequency recognition analysis if the time window length is too short. Figure 2 shows that the correlation relationship between the signal frequency and the reference frequency based on ICCA. 

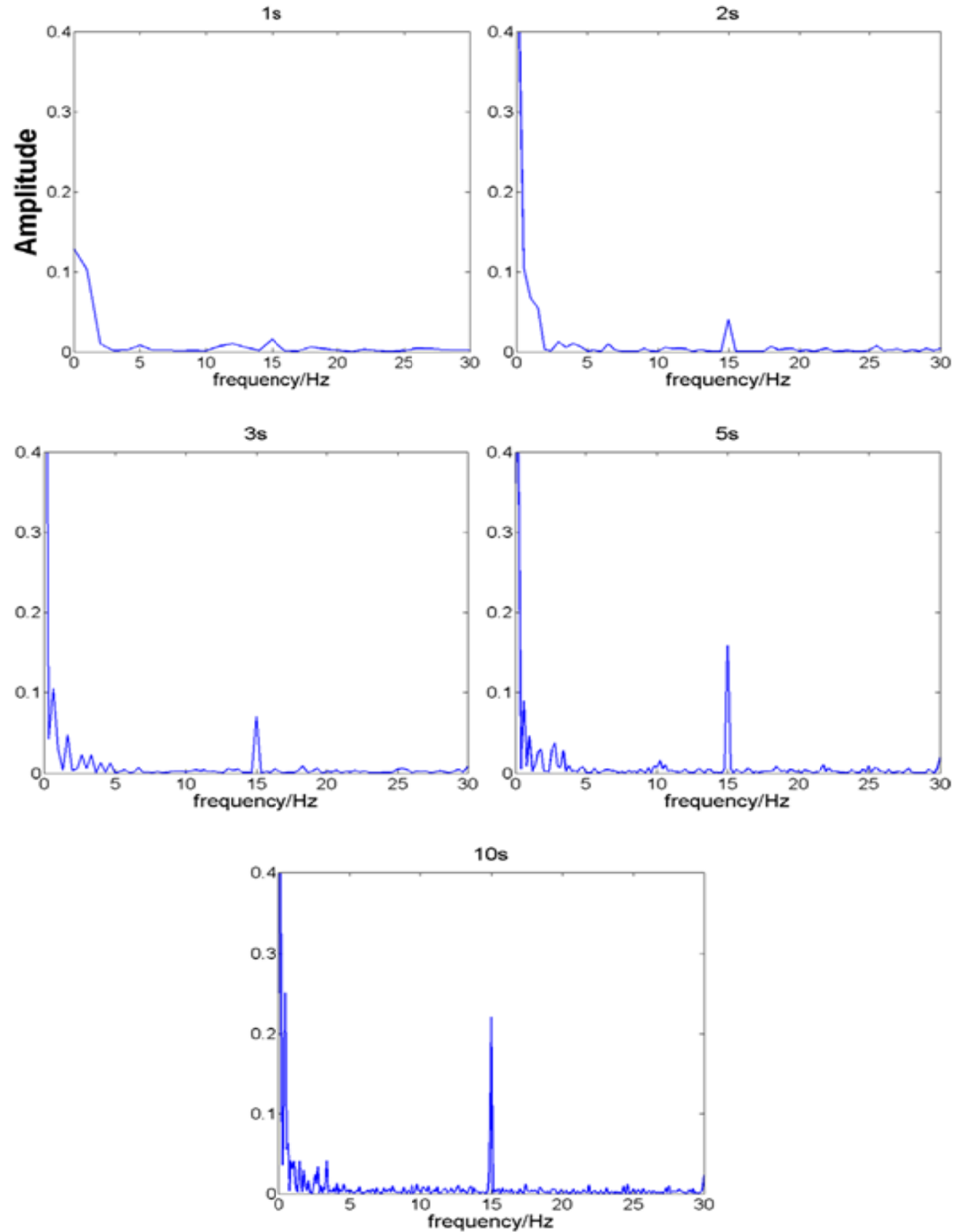

Fig. 1. Power spectrum for different time length with $15 \mathrm{~Hz}$ stimulus for one subject (LX) based on PM. The PM method has significant influences on the accuracy of frequency recognition of SSVEP. 

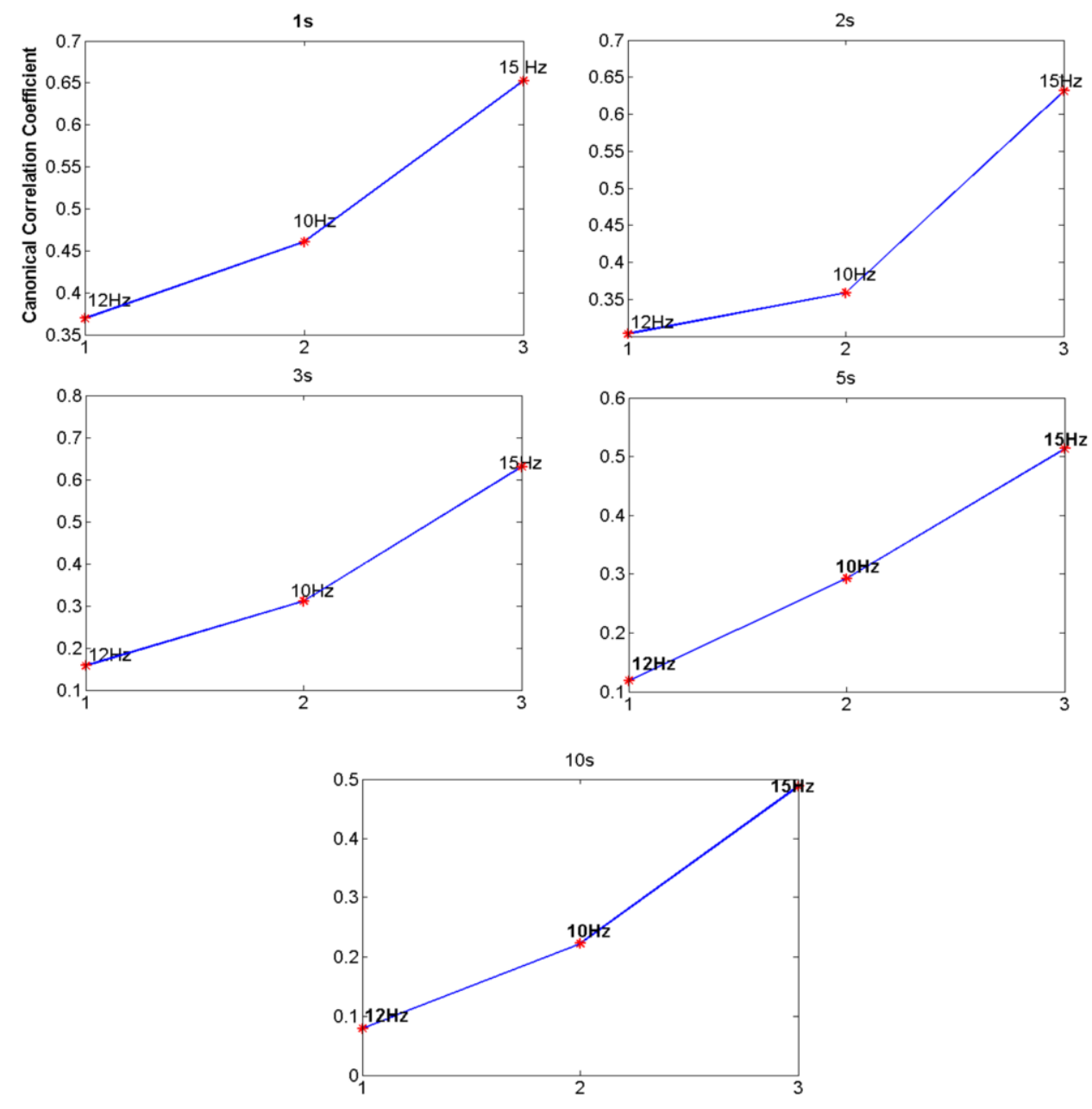

Fig. 2. The correlation relationship between the signal frequency and the reference frequency based on ICCA. The signal frequency recognition depended on the maximum correlation coefficient with the reference signal for one subject (LX).

\section{Discussion and conclusion}

In the present study, two methods, i.e. ICCA and the periodogram method (PM), were employed and compared for frequency recognition of SSVEP recordings. It is observed that compared with PM method ICCA algorithm is able to exhibit higher $(p<0.05)$ recognition accuracy. Further quantitative analysis indicated that the ICCA only need relatively short span for effectively extracting the frequency features in SSVEP measurements. These finding suggests that ICCA is more suitable for frequency recognition of SSVEP recordings than PM. 


\section{Acknowledgement}

This research is supported by SRG2013-00035-FHS Grant and MYRG2014-00093-FHS Grant from University of Macau in Macau. This study is also supported by the National Nature Science Foundation of China (\#31100745, \#61175117, \#91232725), the program for New Century Excellent Talents in University (\#NCET-12-0089), the 863 project 2012AA011601, the CQCBE Project KJ110502, and the undergraduate training programs for innovation and entrepreneurship (201410617003).

\section{References}

[1] D. Regan, Human Brain Electrophysiology: Evoked Potentials and Evoked Magnetic Fields in Science and Medicine, Elsevier Press, Michigan, 1989, pp. 121-150.

[2] F.B. Vialatte, M. Maurice, J. Dauwels and A. Cichocki, Steady-state visually evoked potentials: Focus on essential paradigms and future perspectives, Prog. Neurobiol. (2010), 90418-90438.

[3] O. Friman, I. Volosyak and A. Graser, Multiple channel detection of steady-state visual evoked potentials for braincomputer interfaces, IEEE Trans. Biomed. Eng. 54 (2007), 742-750.

[4] M.A. Lopez, A. Praetor and F. Playa, Use of phase in brain-computer interfaces based on steady-state visual evoked potentials, Morillas Neural Process Lett. 32 (2010), 1-9.

[5] A. Luo and T.J. Sullivan, A user-friendly SSVEP-based brain-computer interface using a time domain classifier, J. Neural Eng. 7 (2010), 1-10.

[6] Z.L. Lin, C.S. Zhang, W. Wu et al., Frequency recognition based on canonical correlation analysis for SSVEP-based BCIs, IEEE Trans. Biomed. Eng. 54 (2007), 1172-1176.

[7] P.L. Nunez, R.B. Silberstein, Z. Shi, M.R. Carpenter, R. Srinivasan, D.M. Tucker, S.M. Doran, P.J. Cadusch and R.S. Wijesinghe, EEG coherency II: Experimental comparisons of multiple measures, Clin. Neurophysiol. 110 (1999), 46986.

[8] D. Yao, A method to standardize a reference of scalp EEG recordings to a point at infinity Physiological measurement 22 (2001), 693-711.

[9] Y. Tian and D. Yao, Why do we need to use a zero reference? Reference influences on the ERPs of audiovisual effects, Psychophysiology 50 (2013), 1282-1290.

[10] Y. Tian, W. Ma, C. Tian et al., Brain oscillations and electroencephalography scalp networks during tempo perception, Neuroscience Bulletin 29 (2013), 731-736.

[11] Y. Qin, P. Xu and D. Yao, A comparative study of different references for EEG default mode network: The use of the infinity reference, Clinical Neurophysiology 121 (2010), 1981-1991.

[12] P. Xu, X. Xiong, Q. Xue, Y. Tian, Y. Peng, R. Zhang, P. Li, Y. Wang and D. Yao, Recognizing mild cognitive impairment based on network connectivity analysis of resting EEG with zero reference, Physiological Measurement, 2014. (in press)

[13] Y. Zhang, P. Xu, D. Guo et al., Prediction of SSVEP-based BCI performance by the resting-state EEG network, Journal of Neural Engineering 10 (2013), 066017.

[14] P. Stoica and R.L. Moses, Introduction to spectral analysis, in: Upper Saddle River, Prentice Hall, New Jersey, 1997, pp. $1-20$. 